\title{
Performance Analysis of Energy Detection Method in Spectrum Sensing Using Static \& Variable Threshold Level for 3G/4G/VoLTE
}

\author{
Prajwal Patil ${ }^{1}$, Pradeep R Pawar ${ }^{2}$, Praneeth P Jain ${ }^{3}$, Manoranjan K V ${ }^{4}$, Devasis Pradhan ${ }^{5 *}$ (IEEE Member)
}

${ }^{1,2,3,4}$ Final Year U.G Students, Department of Electronics \& Communication Engineering, Acharya Institute of Technology, Bengaluru 560107,Karnataka,India

${ }^{5}$ Assistant Professor, Department of Electronics \& Communication Engineering, Acharya Institute of Technology, Bengaluru -560107, Karnataka, India

DOI: $10.36348 /$ sjet.2020.v05i04.007

| Received: 17.04.2020 | Accepted: 25.04.2020 | Published: 29.04.2020

*Corresponding author: Devasis Pradhan

\section{Abstract}

Cognitive radio network is a key to effectively utilize the underutilized radio spectrum. It works on the basis of Software Defined Radio. Spectrum sensing is the most important part of the cognitive radio system and it is used to detect the presence of signal in the air. There are many techniques to sense spectrum like Matched filter based detection, Energy detection, Cyclostationary detection and Wavelet based detection. In the proposed work, Energy detection is chosen in which spectrum sensing is done by considering static and dynamic threshold level. To evaluate the performance of the detection techniques, MATLAB software has been used for simulation. Simulations were carried out and graphs of probability of detection vs. the probability of false alarm were observed and analysed. The detection probability increases with respect to the increase in false alarm. Also significant reductions in probability of missed detection have been achieved with this sensing technique. The detection probability also varies with the SNR value. SNR has a great influence on the probability of detection. With an increase in SNR value, the probability of detection increases.

Keywords: Cognitive Radio, Energy Detection, Probability of Detection, Probability of False Alarm and Receiver Operating Characteristics (ROC).

Copyright @ 2020: This is an open-access article distributed under the terms of the Creative Commons Attribution license which permits unrestricted use, distribution, and reproduction in any medium for non-commercial use (NonCommercial, or CC-BY-NC) provided the original author and source are credited.

\section{INTRODUCTION}

The resources accessible by the existing technologies do not allow them to meet the demand. In order to overcome the scarcity of frequencies, we have new concepts of sharing and sensing of resources, as the dynamic allocation of a radio channel has a new communication has been developed. In the last twenty years have seen a veritable explosion of telecommunication services, from mobile technology to wireless transmission of data, the quantity of general public services increases and the scarcity of frequencies are more than ever aggravated. Cognitive Radio (CR) is a system/model for wireless communication. It is built on software defined radio for Personal Communication Services (PCS). It uses the methodology of sensing and learning from the environment and adapting to statistical variations in real time. The network or wireless node changes its transmission or reception parameters to communicate efficiently anywhere and anytime avoiding interference with licensed or unlicensed users for efficient utilization of the radio spectrum.
The issue of spectrum underutilization in wireless communication can be solved in a better way using Cognitive Radio. Cognitive Radio is characterized by the fact that it can adapt according to the environment by changing its transmitting parameters, such as modulation, frequency, frame format, etc. The main challenges with cognitive radios are that it should not interfere with the licensed users and should vacate the band when required. For this it should sense the signals faster. This work focuses on the spectrum sensing techniques that are based on primary transmitter detection. In this category, four major spectrum sensing techniques "energy detection", "matched filter detection", "cyclostationary feature detection" and "Wavelet detection are addressed. This paper involves the performance analysis of energy detection using fixed and dynamic threshold level for efficient working of cognitive radios in recent generation of communication such as $3 \mathrm{G} / 4 \mathrm{G} / \mathrm{VoLTE}$.

\section{Cognitive Radio (CR)}

The Cognitive radio is a radio that modify to the conditions of the environment by analyzing, 
observing and learning. The cognitive network makes use of this modification for future decisions. CR network is basically used for maximum utilization of the radio bandwidth. The core of the performance optimization is the cognitive process which is shared by the cognitive radio and the cognitive networks. The main part of this process is to learn from the previous decisions and make use of it for future decision.

One of the most important components of the cognitive radio concept is the capability to measure, sense, learn, and be vigilant of the distinguishing feature related to the radio channel attribute, availability of spectrum and power, radio's operating environment, user requirements and applications, available infrastructures and nodes, local policies and other operating limitation. In cognitive radio nomenclature, primary users can be defined as the users who have greater priority on the utilization of a specific part of the spectrum. On the other hand, secondary users, which have lower priority, use this spectrum in such a way that they do not cause interference to primary users as shown in Fig.1.

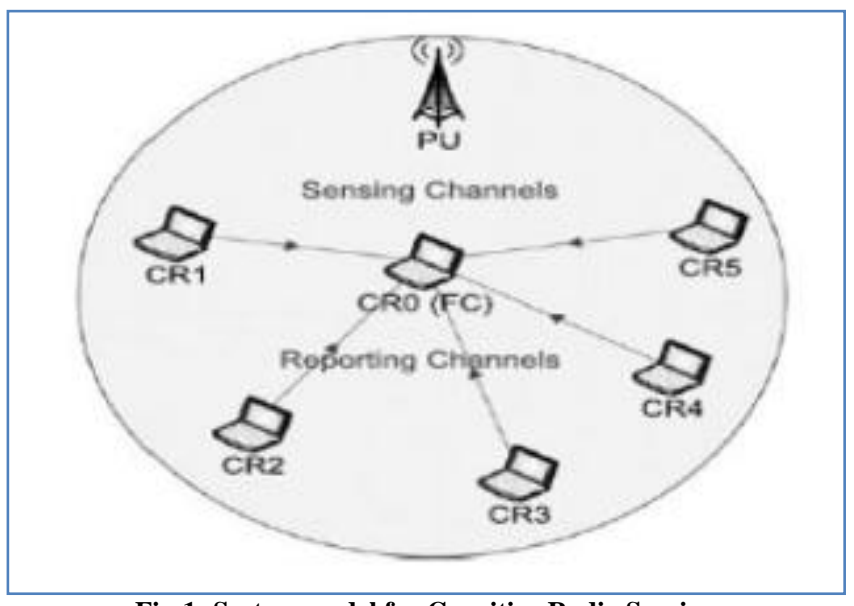

Fig-1: System model for Cognitive Radio Sensing

Therefore, secondary users need to have cognitive radio capabilities, such as sensing the spectrum reliably to check whether it is being used by a primary user and to change the radio parameters to use the unused part of the spectrum. In CR, three leading cognitive tasks are critical: sensing analysis, spectrum analysis, and transmission power control, spectrum management. With the three tasks, the unlicensed users can obtain knowledge of the ecosystem and accordingly fit their spectrum access strategy so as to achieve desired performance and protect the licensed users as much as possible.

\section{Main Function of CR}

i. Spectrum Sensing: It is a fundamental function in $\mathrm{CR}$ to enable cognitive radio users (CRs) to detect the underutilized spectrum of primary systems and improve overall spectrum efficiency.

ii. Spectrum Management: It is required for CR to achieve users' communication needs by capturing the best available spectrum; CR should decide on the best spectrum band and the channels within it to meet the QoS requirements over all available spectrum channels.

iii. Spectrum Mobility: In this is the process whereby cognitive radio users change their frequency

of operation. Cognitive radio networks aim to use the spectrum dynamically by allocating the radio terminals to operate in the greatest available frequency channels.

iv. Spectrum Sharing: The main challenges in open spectrum usage, providing efficient and fair dynamic spectrum allocation methods to distribute the unoccupied spectrum of primary users to the competitive secondary users.

\section{Spectrum Sensing}

Spectrum sensing is the first step in a cognitive radio cycle. It is classified into three primary methods being Non-cooperative spectrum sensing (transmitter detection), Cooperative spectrum sensing and Interference-based spectrum sensing. Three schemes are generally used for the transmitter detection. Spectrum sensing allows the cognitive radio users to learn about the radio environment by detecting the presence of an event using one or multiple sensors. It consists in detecting the PU signal transmission in a given time to make decision to transmit in a frequency band. The spectrum sensing model can be formulated as follows:

\section{$y(n)=w(n) \quad H_{0}: P \boldsymbol{U}$ absent}

$y(n)=h * s(n)+w(n) \quad H_{1}:$ PU present

where, $n=1 \ldots N, N$ is the sample number, $y(n)$ is the SU received signal, $s(n)$ is the PU signal, $w(n)$ is the additive white Gaussian noise (AWGN) with zero mean and variance $\sigma_{\mathrm{n}}{ }^{2}, h$ is the complex channel gain of the sensing channel, $\mathrm{H}_{0}$ denotes the PU signal is absent, and $\mathrm{H}_{1}$ denotes the PU signal is present. 


\section{Energy Detection}

It is a non coherent and non cooperative detection method that detects the primary signal based on the sensed energy. Due to its simplicity and no requirement on a priori knowledge of primary user signal, energy detection (ED) is the most popular sensing technique in cooperative sensing. The block diagram for the energy detection technique is shown in the Fig.2.

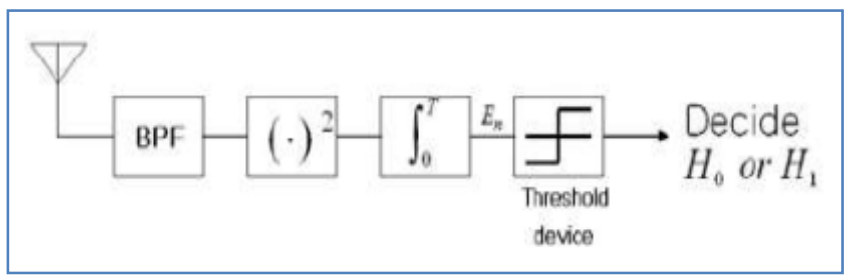

Fig-2: Block Diagram for Energy Detector

In this method, signal is passed through band pass filter of the bandwidth $\mathrm{W}$ and is integrated over time interval. The output from the integrator block is then compared to a predefined threshold. This comparison is used to discover the existence of absence of the primary user. The threshold value can set to be fixed or variable based on the channel conditions.

Energy Detection is the most common way of spectrum sensing because of its low computational and implementation complexities. It is a more generic method as the receivers do not need any knowledge on the primary user's signal. The signal is detected by comparing the output of the energy detector with a threshold which depends on the noise floor. The received signal sample of a secondary user can be represented as

$y(n)=w(n) \quad H_{0}: P U$ absent........ (i)
$y(n)=h * s(n)+w(n) \quad H_{1}:$ PU present....(ii)

Where $\mathrm{n}$ denotes the sample index, $\mathrm{h}(\mathrm{n})$ denotes the impulse response of the channel between the primary and secondary users, $s(n)$ is the signal from the primary user, $\mathrm{w}(\mathrm{n})$ denotes zero-mean additive white Gaussian noise and $\mathrm{H}_{0}$ and $\mathrm{H}_{1}$ represent hypothesis corresponding to the absence and presence of the primary user's signal, respectively. We consider the use of energy detection for the spectrum sensing. Then, the probability of false alarm and probability of detection for the energy detector can be represented as

$P_{f a}=Q\left[\frac{\gamma-\sigma_{n}^{2}}{\sqrt{2 \sigma_{n}^{4} / N}}\right] \ldots \ldots . . . .($ iii $)$

$P_{d}=Q\left[\frac{\gamma-\left(p+\sigma_{n}^{2}\right)}{\sqrt{2\left(p+\sigma_{n}^{2}\right) / N}}\right] \ldots . .$. (iv)

$N=\left[Q^{-1}\left(P_{f a}\right)-Q^{-1}\left(P_{d}\right) \sqrt{2 \gamma+1}\right]^{2} \gamma^{-2}$

Where $\mathrm{N}$ is the number of samples, $\mathrm{Q}$ is the standard Gaussian complementary cumulative distribution

function, $p$ is the average signal power, $\sigma_{n}^{2}$ is the noise variance and $\gamma$ is the threshold level to be determined.
The output $\gamma$ of the detector is compared to a threshold to make the right decision:

If $\gamma \geq$ threshold then PU signal is present.

If $\gamma<$ threshold then PU signal is absent.

Where is defined as

$\gamma=F^{-1}\left(1-P_{f a} \mid U, \sigma_{\mathrm{n}}^{2}\right)$

The important challenge with the energy detector based sensing is the selection of the threshold for detecting primary users. The other challenges include inability to differentiate interference from primary users and noise and poor performance under low signal-to-noise ratio values. $\mathrm{P}_{\mathrm{d}}$ (probability of detection) and $\mathrm{P}_{\mathrm{fa}}$ (probability of false alarm) are the important factors for energy based detection which gives the information of the availability of the spectrum.

\section{Decision Threshold}

The decision threshold and the noise uncertainty (in turn the SNR and SNR Wall) are key parameters in the performance of the energy detector. The value of the decision threshold $\lambda$ can be chosen for an optimal compromise between $\mathrm{P}_{\mathrm{fa}}$ and $\mathrm{P}_{\mathrm{d}}$. This method requires prior knowledge about the noise and detected signal power, both of which are not easy to determine.

Detected signal power is difficult to estimate primarily because of transmission and propagation characteristics which include fading possibly due to user movement and interference. Setting the threshold just above the noise floor is typically employed for fixed threshold purposes, but this is not ideal since noise can change due to various reasons.

Noise power is primarily due to white noise that varies with temperature fluctuations, inefficient low noise amplifiers in receivers and filters, or leakage of signals with small variations. High noise power that leads to low SNR values can cause sharp degradations in the energy detector performance due to the SNR wall. 
The energy detector's limitation is that it cannot differentiate signal types but can only determine the presence of the signal; therefore, the energy detector is prone to the false detection triggered by the unintended signals. The $\mathrm{P}_{\mathrm{fa}}$ and $\mathrm{P}_{\mathrm{d}}$ are related to each other through decision regions and likelihood functions as

$P_{f a}=\int_{R_{1}} p_{X \mid M_{0}}\left(X \mid M_{0}\right) d x \ldots \ldots \ldots \ldots(v i i)$

$$
P_{d}=\int_{R_{1}} p_{X \mid M_{1}}\left(X \mid M_{1}\right) d x \ldots \ldots \ldots \ldots(v i i i)
$$

The decision region $\mathrm{R}_{1}$ which means that when $\mathrm{X}$ (the random variable) lays within the decision region $\mathrm{R}_{1}$ then the hypothesis $\mathrm{H}_{1}$ is selected. The threshold value describes the decision regions where $P_{f a}$ is specified for reasonable performance of the CR. The relationship between Pfa. $\mathrm{P}_{\mathrm{d}}$ and the threshold can be shown in Fig. 3 below.

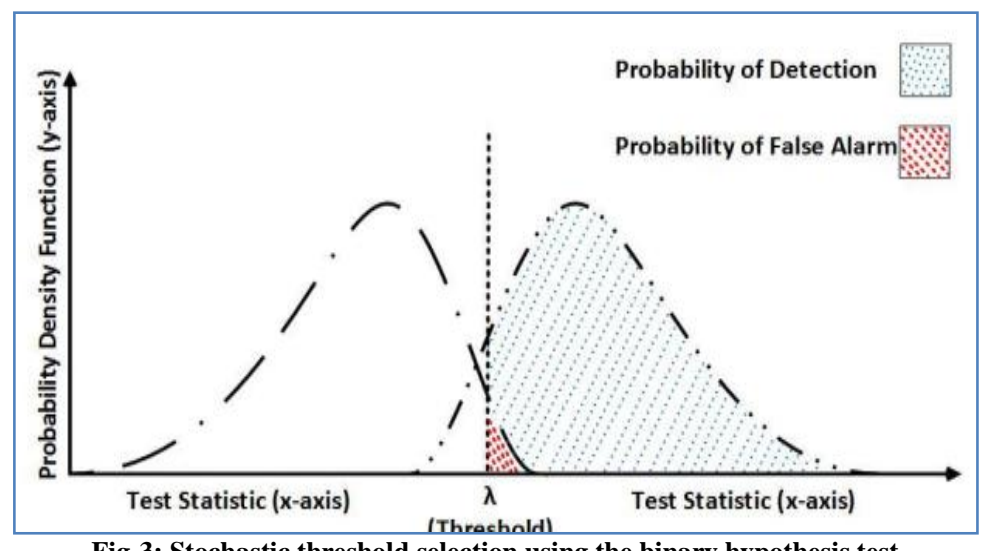

Fig-3: Stochastic threshold selection using the binary hypothesis test

\section{RESULTS AND DISCUSSION}

ROC plots for Energy Detector based spectrum sensing

$\mathrm{Pd}=$ Probability of detection

Pfa $=$ Probability of false alarm

$\mathrm{N}=$ Number of samples

$\mathrm{SNR}=$ Signal to noise ratio

Detection probability $\left(\mathrm{P}_{\mathrm{d}}\right)$ and False alarm probability $\left(\mathrm{P}_{\mathrm{fa}}\right)$ ) are the key measurement metrics that are used to analyze the performance of spectrum sensing techniques. The performance of an spectrum sensing technique is illustrated by the receiver operating characteristics (ROC) curve which is a plot of $P_{d}$ versus $\mathrm{P}_{\mathrm{fa}}$. The performance of energy detector is analyzed using ROC (Receiver operating characteristics) curves. Monte-Carlo method is used for simulation.

\section{First Simulated Result}

The plot of Probability of detection (Pd) versus Signal to Noise Ratio (SNR)

In the simulation, study input random bit stream is multiplied by $1 \mathrm{MHz}$ sinusoidal carrier signal to get $1 \mathrm{MHz}$ BPSK modulated signal, which is transmitted in AWGN channel. The plot of Probability of detection $\left(\mathrm{P}_{\mathrm{d}}\right)$ and Signal to Noise Ratio (SNR) is illustrated in Fig.4. Signal to Noise Ratio (SNR) is on $\mathrm{X}$-axis and probability of detection $\left(\mathrm{P}_{\mathrm{d}}\right)$ is on $\mathrm{Y}$-axis. The detection performance can be performed by varying the SNR from $-15 \mathrm{~dB},-14 \mathrm{~dB} \ldots \ldots \ldots . .0 \mathrm{~dB}$ and finding the probability of detection by using Monte Carlo simulation.

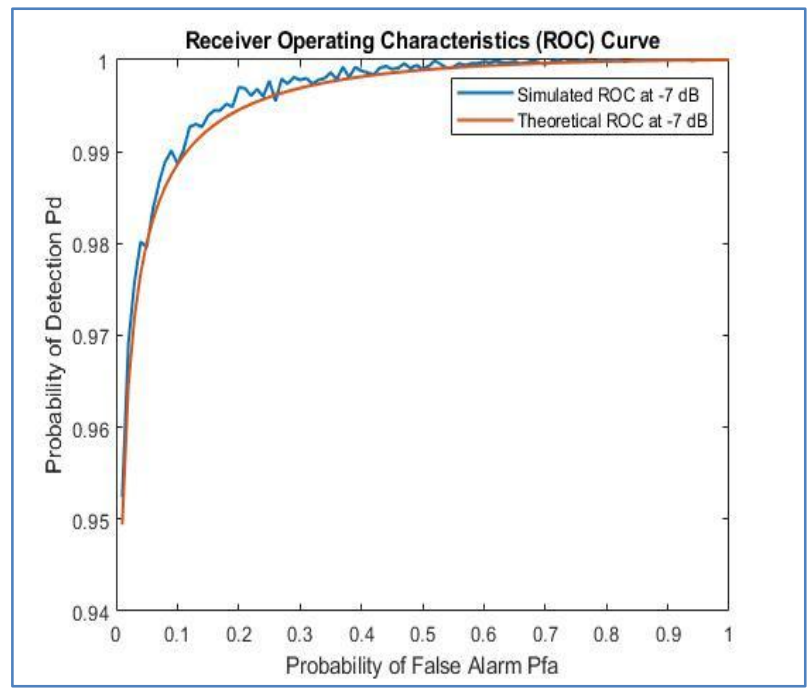

Fig-4: Plot for Pd vs SNR Energy detector based spectrum sensing at $\mathrm{N}=\mathbf{1 0 0 0}$

\section{Second Simulated Result}

The plot of Probability of false alarm versus Probability of detection.The plot of Probability of false alarm versus Probability of detection is illustrated in Fig.5. Probability of false alarm (Pf) is on X-axis and probability of detection $(\mathrm{Pd})$ is on $\mathrm{Y}$-axis. In the simulation, study input random bit stream is multiplied by $1 \mathrm{MHz}$ sinusoidal carrier signal to get $1 \mathrm{MHz}$ BPSK modulated signal, which is transmitted in AWGN channel. The detection performance can be performed by varying the probability of false alarm from $0.01,0.02 \ldots . . .1$ and finding the probability of detection by using Monte Carlo simulation. Here the number of sample points taken is $\mathbf{N = 1 0 0 0}$ and $\mathbf{S N R}=\mathbf{- 7} \mathbf{~ d B}$. 


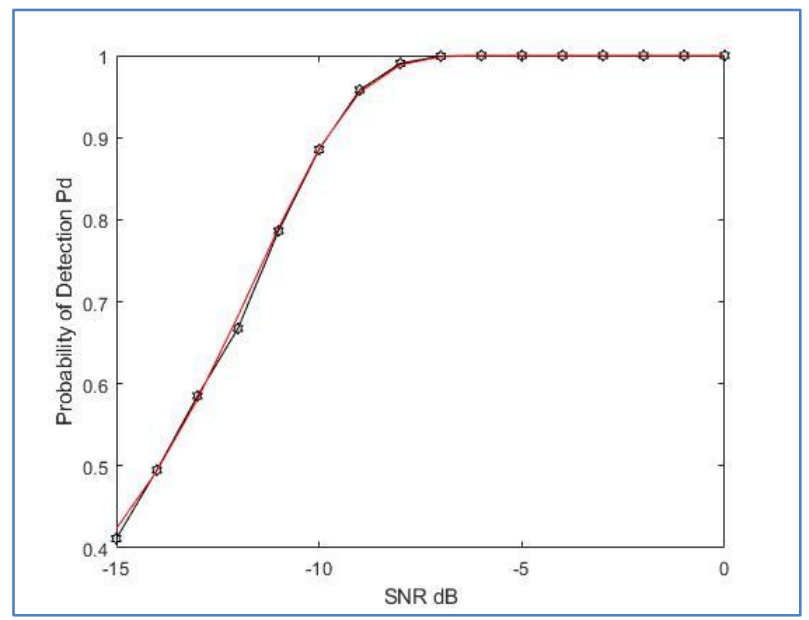

Fig-5: ROC curve for Pf vs Pd Energy detector based spectrum sensing at $\mathrm{SNR}=-7 \mathrm{~dB}$

\section{Third Simulated Result}

The plot of Probability of false alarm versus Probability of detection for constant and variable threshold energy detector.

A MATLAB simulation of the ROC curves for the constant and proposed variable threshold energy detectors was carried out. It was expected that the ROC curves of the constant threshold and variable threshold would be different with all parameters the same. A higher $\mathbf{P}_{\mathbf{d}}$ for each $\mathbf{P}_{\mathbf{f a}}$ in the variable threshold energy detector plot of each ROC curve was expected. This was generally the case from the MATLAB simulation at low SNR values Fig.6 shows the ROC curve plots for both thresholds.

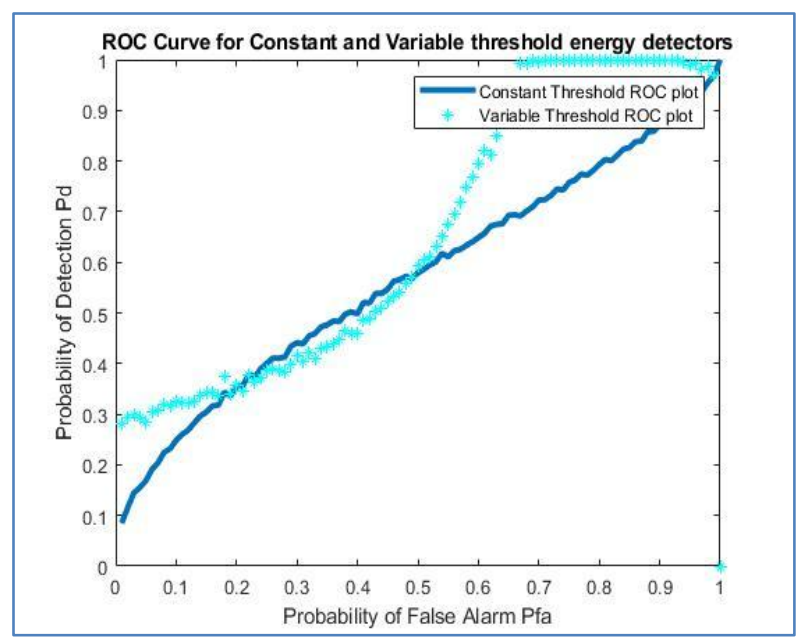

Fig-6: ROC curve plots for both thresholds

\section{CONCLUSION}

Spectrum is a very valuable resource in wireless communication systems, and it has been a focal point for research and development efforts over the last several decades. Cognitive radio, which is one of the efforts to utilize the available spectrum more efficiently through opportunistic spectrum usage, has become an exciting and promising concept. In this paper, we examined various aspects of cognitive radio and identified spectrum sensing as the prerequisite requirement for the deployment of cognitive radio oriented wireless networks.

ED requires knowledge of the noise variation only. The ED spectrum sensing method albeit presents benefits that include no need for knowledge of enough relevant information about the PU such as the modulation type or transmit power, does not perform well in low SNR environments. The detection probability also varies with the SNR value. SNR has a great influence on the probability of detection. With an increase in SNR value, the probability of detection increases and we get $\mathrm{SNR}=-7 \mathrm{~dB}$, where we get a detection probability of 1 . Hence, we almost obtain the final result on energy detection according to our expectation.

\section{ACKNOWLEDGMENT}

Firstly, we highly indebted to the Department of Electronics \& Communication Engineering for giving us the opportunity to complete this research work. We sincerely express our sincere gratitude and deepest appreciation to Dr. Rajeswari, Professor, HoD of ECE and Principal of Acharya Institute of Technology, Bangalore, Karnataka, for guiding and encouraging us to develop and simulate the control designs essential for this work and support. We would like thank to Mr. Devasis Pradhan for guiding us throughout the course of our project through which the research work come into a streamline work and we also thankful to our beloved parents for their trust on us and their believe.

\section{REFERENCE}

1. Jain, P. P., Pawar, P. R., Patil, P., \& Pradhan, D. (2019). Narrowband Spectrum Sensing in Cognitive Radio: Detection Methodologies.

2. Devasis, P., \& Priyanka, K.C. (2019). "Effectiveness of Spectrum Sensing in Cognitive Radio toward 5G Technology", Saudi Journal of Engineering and Technology, Dec. 4(12): 473-785

3. Wang, B., \& Liu, K. R. (2010). Advances in cognitive radio networks: A survey. IEEE Journal of selected topics in signal processing, 5(1), 5-23.

4. Quan, Z., Cui, S., Sayed, A. H., \& Poor, H. V. (2008). Optimal multiband joint detection for spectrum sensing in cognitive radio networks. IEEE transactions on signal processing, 57(3), 1128-1140.

5. Ejaz, W., ul Hasan, N., Lee, S., \& Kim, H. S. (2013). I3S: Intelligent spectrum sensing scheme for cognitive radio networks. EURASIP Journal on Wireless Communications and Networking, 2013(1), 26.

6. Umar, R., \& Sheikh, A. U. (2013). A comparative study of spectrum awareness techniques for cognitive radio oriented wireless networks. Physical Communication, 9, 148-170. 
7. Benmammar, B., Amraoui, A., \& Krief, F. (2013). A survey on dynamic spectrum access techniques in cognitive radio networks.

8. Joo, C., \& Shroff, N. B. (2017, May). A novel coupled queueing model to control traffic via QoSaware collision pricing in cognitive radio networks. In IEEE INFOCOM 2017-IEEE Conference on Computer Communications (pp. 1-9). IEEE.

9. Lu, Q., Yang, S., \& Liu, F. (2017). Wideband spectrum sensing based on riemannian distance for cognitive radio networks. Sensors, 17(4), 661.

10. Fihri, W. F., Arjoune, Y., El Ghazi, H., Kaabouch, N., \& El Majd, B. A. (2018, January). A particle swarm optimization based algorithm for primary user emulation attack detection. In 2018 IEEE 8th Annual Computing and Communication Workshop and Conference (CCWC) (pp. 823-827). IEEE.

11. Gao, Y., Chen, Y., \& Ma, Y. (2017). SparseBayesian-learning-based wideband spectrum sensing with simplified modulated wideband converter. IEEE Access, 6, 6058-6070.

12. Yucek, T., \& Arslan, H. (2009). A survey of spectrum sensing algorithms for cognitive radio applications. IEEE communications surveys \& tutorials, 11(1), 116-130.

13. Eduardo, A. F., \& Caballero, R. G. G. (2015, May). Experimental evaluation of performance for spectrum sensing: Matched filter vs energy detector. In IEEE Colombian Conference on Communication and Computing (IEEE COLCOM 2015) (pp. 1-6). IEEE.

14. Salahdine, F., El Ghazi, H., Kaabouch, N., \& Fihri, W. F. (2015, October). Matched filter detection with dynamic threshold for cognitive radio networks. In 2015 international conference on wireless networks and mobile communications (WINCOM) (pp. 1-6). IEEE.
15. Bagwari, A., \& Singh, B. (2012, November). Comparative performance evaluation of spectrum sensing techniques for cognitive radio networks. In 2012 Fourth International Conference on Computational Intelligence and Communication Networks (pp. 98-105). IEEE.

16. Taruna, S., Pahwa, B., \& Kaur, I. (2014, February). Assessment of energy detection spectrum sensing under different wireless channels. In 2014 IEEE International Advance Computing Conference (IACC) (pp. 276-281). IEEE.

17. Omer, A. E. (2015, September). Review of spectrum sensing techniques in Cognitive Radio networks. In 2015 International Conference on Computing, Control, Networking, Electronics and Embedded Systems Engineering (ICCNEEE) (pp. 439-446). IEEE.

18. Digham, F. F., Alouini, M. S., \& Simon, M. K. (2003, May). On the energy detection of unknown signals over fading channels. In IEEE International Conference on Communications, 2003. ICC'03. 5, 3575-3579).

19. Akyildiz, I. F., Lee, W. Y., Vuran, M. C., \& Mohanty, S. (2006). NeXt generation/dynamic spectrum access/cognitive radio wireless networks: A survey. Computer networks, 50(13), 2127-2159.

20. Shaikh, A. Z., \& Altaf, T. (2012). Performance analysis of correlated multiple antenna spectrum sensing cognitive radio. International Journal of Computer Applications, 50(5).

21. Oh, D. C., \& Lee, Y. H. (2009). Energy detection based spectrum sensing for sensing error minimization in cognitive radio networks. International Journal of Communication Networks and Information Security (IJCNIS), 1(1), $1-5$. 